\title{
Retrieval of Separated Instrument by Trephining Technique in Radix Entomolaris - A Case Report
}

\author{
Sumaiya Dossa ${ }^{1}$ \\ Javeria Ali Khan ${ }^{2}$ \\ Arshad Hasan ${ }^{3}$
}

\author{
BDS \\ BDS, FCPS \\ BDS, FCPS
}

The endodontic treatment of a tooth with radix entomolaris can present significant challenges due to the presence of root curvature. The present report describes the endodontic treatment of a permanent mandibular first molar with type II radix entomolaris and separation of endodontic file in the root canal followed by its retrieval using the trephining technique. A 38 years old female patient visited the Department of Operative Dentistry at the Dow University of Health Sciences with complain of spontaneous and lingering pain and food impaction in tooth \#46 since six months. On intraoral examination, tooth \#46 had class II cavity. On periapical radiograph, an accessory root was observed. Endodontic treatment of the tooth was initiated. During shaping of the accessory canal, SX separated. The separated file was bypassed and successful retrieval was performed using the trephining technique. Endodontic treatment of the tooth was completed. The tooth was found to be asymptomatic in the followup visits and the patient was referred for a full coverage crown.

KEY WORDS: Radix entomolaris, trephining, Protaper Universal, modified gates glidden, root canal curvature, cyclic fatigue. HOW TO CITE: Dossa S, Khan JA, Hasan A. retrieval of separated instrument by trephining technique in radix entomolaris - a case report. J Pak Dent Assoc 2019;28(3):143-148.

DOI: https://doi.org/10.25301/JPDA.283.143

Received: 31 January 2019, Accepted: 10 March 2019

\section{INTRODUCTION}

$\mathrm{K}$ nowledge of the normal anatomy and variations in the anatomy of the root canal system is important to perform good quality endodontic treatment. Normal root canal anatomy of permanent mandibular first molars consists of three root canals; two mesial canals and one distal canal. ${ }^{1}$ Variations in the root canal anatomy of mandibular first molars is a common finding. ${ }^{2,3}$ A number of such anatomical variations have been reported one of which is the supernumerary root which, in the mandibular first molars, was first reported by Carabelli in $1884 .{ }^{4}$ The supernumerary root on the lingual surface of mandibular first molars was named as radix entomolaris by Mihaly Lenhossek in $1922 .{ }^{5}$

The endodontic treatment of a tooth with Radix entomolaris can present significant challenges due to the presence of root curvature. Ribeiro \& Consolaro classified radix entomolaris into type I, II and III according to the

1. MDS Trainee, Department of Operative Dentistry, Dow Dental College, Dow University of Health Sciences.

2. Assistant Professor, Department of Operative Dentistry, Dow Dental College, Dow University of Health Sciences.

3. Professor, Department of Operative Dentistry, Dow Dental College, Dow University of Health Sciences. Principal, Dow Dental College, Dow University of Health Sciences.

Corresponding author: "Dr. Sumaiya Dossa" < sumaiya_aquarian@yahoo.com > buccolingual curvature. ${ }^{6}$ Root canal instrumentation of a curved canal with stainless steel files can produce errors such as ledges, zips, elbows, apical transportation, loss of working length, or perforations. ${ }^{7}$ The Nickel-titanium endodontic files can reduce the incidence of above mentioned errors, as they are more flexible in the canal curvature. However, these files can undergo unexpected fracture as a result of cyclic fatigue. ${ }^{8}$

The present report describes the endodontic treatment of a permanent mandibular first molar with radix entomolaris and separation of endodontic file in the root canal followed by its retrieval using the trephining technique.

\section{REPORT}

A 38 years old female patient visited the Department of Operative Dentistry at the Dow University of Health Sciences with complain of pain and food impaction in tooth \#46 since six months. According to the patient, pain started spontaneously from the right side of the lower jaw and lingered on until medicine was taken for pain relief. Moreover, she also reported of pain radiation to the ipsilateral side of the head. Aggravating factors for pain included hot and cold meals and food impaction and the pain was relieved by analgesics. 
The family history, history of hospitalization and dental history were not significant. Her habits included tooth brushing once daily with fluoridated toothpaste, frequent intake of sweets not followed by tooth brushing or mouth rinsing and betel nut intake in childhood.

On extraoral examination and intraoral soft tissue examination, no significant findings were observed. Upon dental examination, it was observed that the patient had susceptible pits and fissures in all lower molars. Third molars were not present in the oral cavity. Tooth \#46 had class II cavity. The tooth was not tender to percussion or palpation. There were no broken down roots; filled, mobile or traumatized teeth. Probing depths of all teeth were recorded by using Michigan probe and were found to be within normal range except for the distobuccal and distolingual pocket depths of the right mandibular first molar which were found to be $3.5 \mathrm{~mm}$. Bleeding on probing was also observed on the same locations.

Two periapical radiographs of the right mandibular molars were recorded. The second radiograph (figure 1) was recorded with a slight cone shift (approximately 20 degrees) in the horizontal plane. A class II cavity on the distal surface

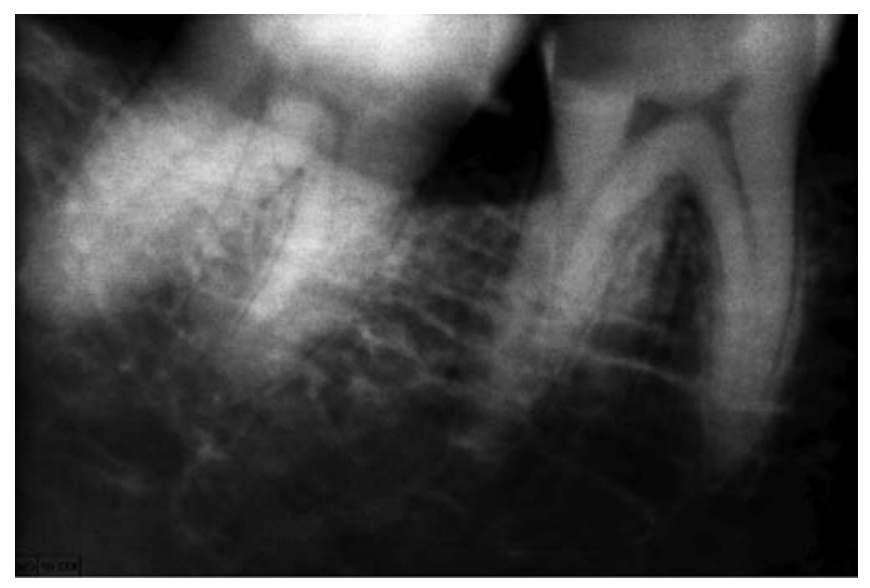

Figure 1: Pre-operative radiograph showing accessory root

of right mandibular first molar was observed in close proximity to the pulp. The periapical area of the same tooth was found to be normal. A supernumerary root was observed in the first molar on second radiograph.

Cold test of tooth \#46 with endo ice (Henry Schein, NY, USA). Patient gave an exaggerated response on cold test. The pain lingered for more than 10 seconds.

Based on the endodontic diagnosis criteria provided by the American Association of Endodontists ${ }^{9}$, tooth \#46 was diagnosed as irreversible pulpitis with normal periapical area.

A general treatment plan consisting of five phases was formed for the full mouth treatment of the patient. The treatment plan consisted of an emergency phase (emergency chamber opening and pulpectomy of the painful tooth), non surgical phase (enforcing oral hygiene instructions, plaque control, scaling and fissure sealants for molars with susceptible fissures), surgical phase (continuation of endodontic therapy of right mandibular first molar), restorative phase (core buildup and porcelain fused to metal crown for the endodontically treated tooth) and maintenance phase (reinforcement of oral hygiene instructions and evaluation of fissure sealants).

Emergency chamber opening of the right mandibular first molar was performed to relieve the pain. The tooth was temporarily restored with Cavit (Meta Biomed Co. Ltd., Korea).

Single tooth isolation was achieved using rubber dam (Henry Schein, NY, USA). Four pulp canal orifices were observed with the distolingual canal orifice positioned slightly offset. The distolingual canal orifice was suspected to be the orifice for accessory root canal of the supernumerary root (radix entomolaris). Canal patency was established using patency files $06 / 0.02$ and $08 / 0.02$ and $10 / 0.02$ (Mani, Inc., Tochegi, Japan). Orifices were then widened with gates gliden number 5 (Mani, Inc., Tochegi, Japan) in a slow speed handpiece such that half of the head of the gates gliden drill was inserted into the orifice while working (figure 2). Pulp chamber and pulp canals were kept moist with sodium hypochlorite ( $\mathrm{NaOCl}) 2.5 \%$.

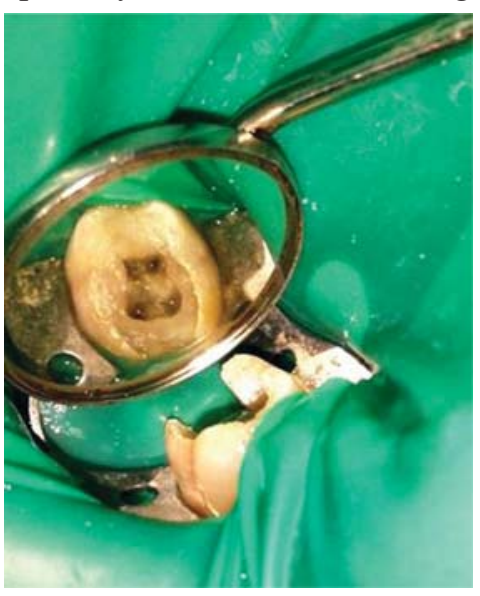

Figure 2: Pulp chamber of the tooth with four canal orifices visible.
Next, the SX file of the rotary Protaper Universal (PTU) (Dentsply Tulsa Dental Specialities, Tulsa, OK) was used in brushing motion for coronal flaring. 17\% EDTA (Meta Biomed Co. Ltd., Korea) was used as lubricant. The file was inserted into the canal in multiple strokes in crown down manner. When the file was taken out of the distolingual canal, it was found to be 3 to $4 \mathrm{~mm}$ shorter in length. A periapical radiograph was recorded and a separated fragment of SX was confirmed in the accessory canal (figure 3). The separated fragment was present in the coronal and middle third of the root. Upon observing the pulp chamber, a silver spot (coronal portion of the separated instrument) was observed in the distolingual canal orifice. The tooth was temporarily filled with Cavit (Meta Biomed Co. Ltd., Korea) and the patient was recalled for an attempt to bypass the separated instrument. 


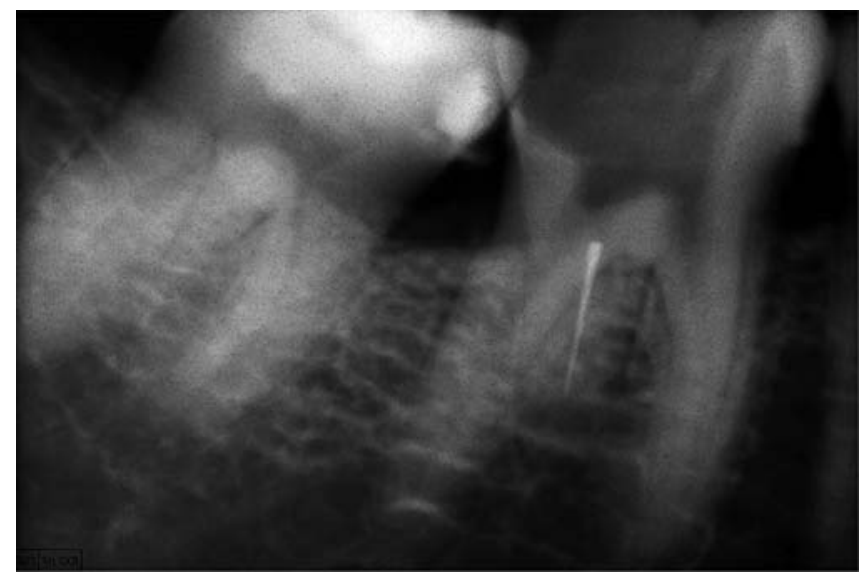

Figure 3: Separated SX in accessory root canal

In the second visit, local anesthesia was administered and single tooth isolation was achieved using rubber dam. Distolingual canal was flared with gates glidden number 4 and 3 till the blocked area. Separated file was bypassed with manual stainless steel $\mathrm{K}$ files $06 / 0.02,08 / 0.02,10 / 0.02$, $15 / 0.02,20 / 0.02,25 / 0.02,30 / 0.02$ and 35/ 0.02 (Mani Inc., Tochegi, Japan). Apical ends of all files used to bypass the separated fragment were bent at acute angle (figure 4). The tooth was temporarily restored.

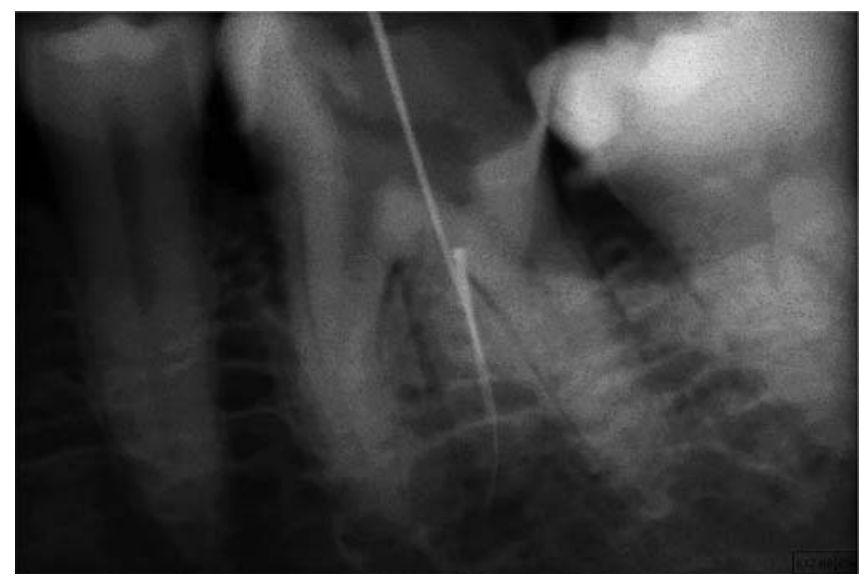

Figure 4: Separated file by passed

In the third visit, local anesthesia was administered and single tooth isolation with rubber dam was achieved. Attempt to retrieve the separated SX file was performed. Gates glidden number 3 was modified by cutting its bud at its maximum cross sectional diameter perpendicular to its long axis (figure 5), as described in a previous article. ${ }^{10} \mathrm{~A}$ short straight fissure diamond bur (Mani Inc., Tochegi, Japan) was used to cut the bud of gates glidden. This modified gates glidden was then used to create a circumferential staging platform. The modified gates glidden was used in a slow speed handpiece (NSK, Japan), carried into the preenlarged canal and directed apically until it lightly contacted the head of

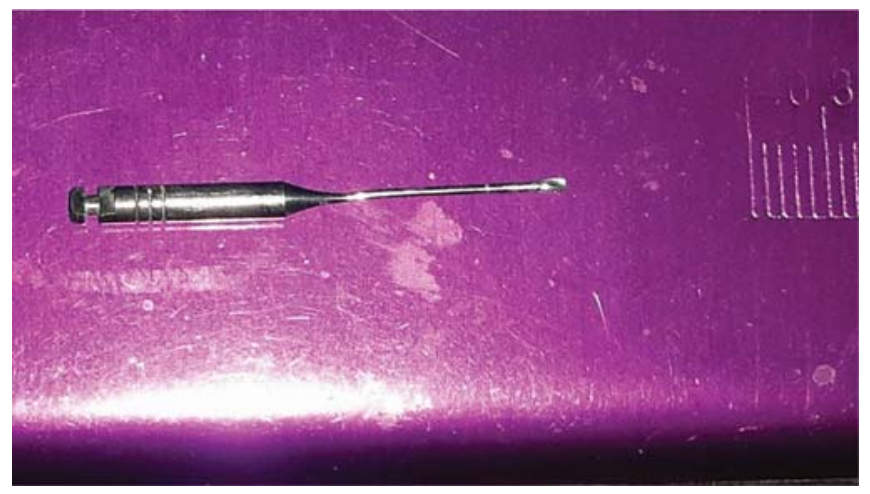

Figure 5: Modified gates glidden no. 3

the separated SX file. After the creation of staging platform the other orifices were covered with cotton pellets and trephining of dentine around the separated SX file was attempted. An ultrasonic scaler tip was used at low power. The ultrasonic tip was placed on the staging platform between exposed end of the separated file and the canal wall and was vibrated around the separated file in a counterclockwise direction. For appropriate visualization of the ultrasonic tip movement, it was used without water in a dry field. However, due to the increased increased thickness and short length of ultrasonic tip, trephining with it was discontinued. A gates glidden number 2 was modified in the same manner as mentioned above and was used for trephining. Approximately $3 \mathrm{~mm}$ of dentine around the head of the separated file was removed to expose it. No loosening of separated file fragment was observed. Since the separated fragment had been bypassed already, an attempt was made to remove the fragment with $\mathrm{H}$ files. $\mathrm{H}$ file number 20/ 0.02, 25/ 0.02 and 30/ 0.02 (Mani, Inc., Tochegi, Japan) were used in sequence in filing (translational in and out) motion which resulted in loosening of the separated fragment. The exposed coronal portion of the separated fragment was grasped with tweezer and removed from the canal (figure 6). Canal was irrigated

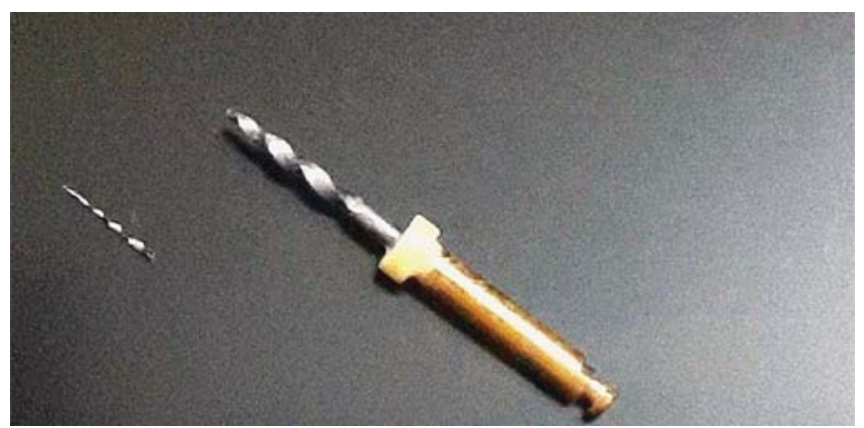

Figure 6: Separated SX retrieved

copiously with $\mathrm{NaOCl}$ and the tooth was temporized. A periapical radiograph was recorded to confirm complete removal of the separated SX file fragment (figure 7). 


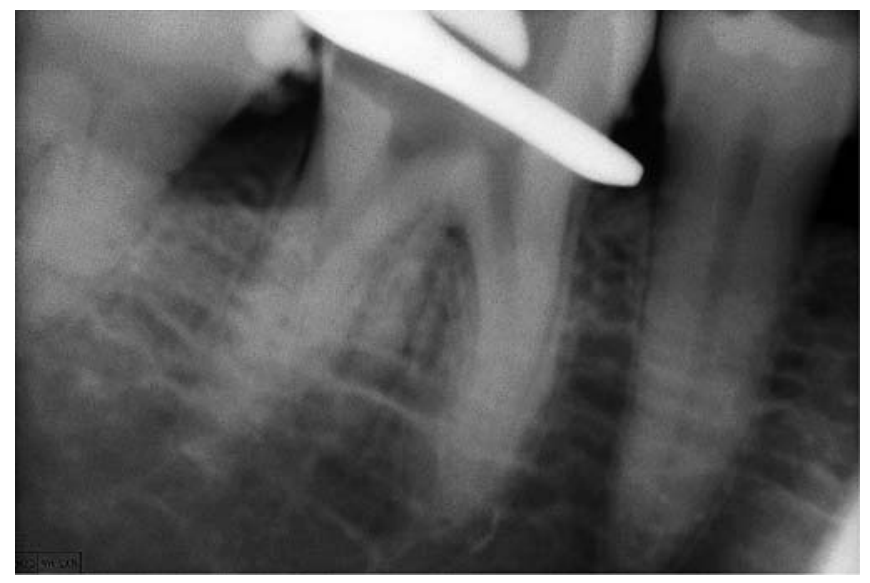

Figure 7: Radiograph confirming successful retrieval of separated file

In the next visit, the tooth was isolated under local anesthesia and cleaning and shaping of all four canals was completed. The mesiobuccal, mesiolingual and distobuccal canals were shaped with rotary Protaper Universal files in an endomotor (X-Smart, Dentsply Maillefer, Ballaigues, Switzerland) at a speed of $250 \mathrm{rpm}$ and torque $2.6 \mathrm{nM}$. Apices of all three canals were prepared till F2. Calcium hydroxide (Ultradent Products Inc., Utah, USA) was placed in all four canals as an intracanal medicament using lentulospiral number 25 (Mani Inc., Tochegi, Japan) in a slow speed handpiece.

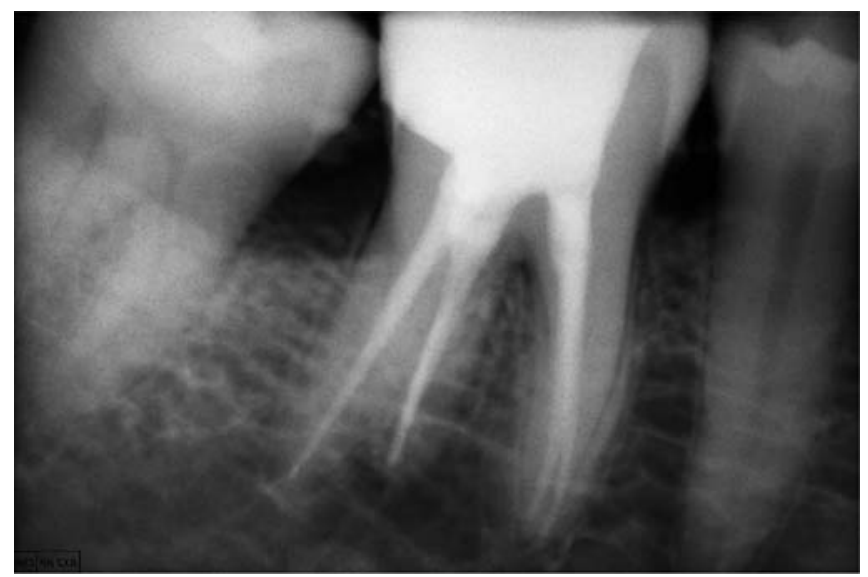

Figure 8: Post-operative radiograph

In the final visit, quadrant isolation was performed under local anesthesia and the calcium hydroxide was removed removed using $0.9 \%$ saline (Frontier Dextrose Ltd., Haripur, Pakistan.) Canals were dried with respective paper points. Calcium hydroxide based sealer Sealapex (Kerr Corporation, USA) was used and the mesiobuccal, mesiolingual and distobuccal canals were obturated using the single cone obturation technique. The accessory root canal was obturated using the lateral condensation technique with number 40 as the master apical gutta percha cone. Excess GP above the orifice levels was seared off. Sectional ivory retainer was placed. The cavity was etched (3M ESPE, USA) for 30 seconds with continuous brushing using a microbrush. Etchant was washed for 15 to 20 seconds. A cotton pellet was placed inside the cavity to dry excess water. Bonding agent (3M ESPE, USA) was applied using microbrush. Gentle air was blown over cavity for 15 seconds and the bonding agent was light cured for 15 seconds. The cavity was then restored with packable composite (3M ESPE, USA) (figure 8).

Followup visit was conducted after one week. The patient was asymptomatic and the tooth was not tender to percussion. Patient was referred for a full coverage crown on the root treated tooth.

\section{DISCUSSION}

Carlsen and Alexandersen classified radix entomolaris into type $\mathrm{A}$, type $\mathrm{B}$, type $\mathrm{C}$ and type $\mathrm{AC}$ according to the position of its cervical portion. ${ }^{11}$ According to the preoperative radiographic evaluation and evaluation of the access cavity, this case would fall into the category of type B i.e. distally located cervical part with one normal distal root component. Ribeiro \& Consolaro classified radix entomolaris into type I, II and III according to the buccolingual curvature. ${ }^{6}$ According to our clinical judgement, this case would be classified as type II as a sharp curvature was felt at the canal entrance while scouting with small manual files. A case report described the Endodontic treatment of type II of radix entomolaris. ${ }^{6}$

Radix entomolaris is mostly superimposed over distal root buccolingually. A radiograph with a 30 degrees shift in horizontal plane makes the accessory root visible. In this case, no accessory root was visible on preoperative periapical radiograph recorded with cone beam parallel to the long axis of the tooth. However, when the cone beam was shifted mesially at an angle of 20 degrees, an accessory root was visible. Application of SLOB principle confirmed the position of accessory root to be lingual.

Due to eccentric location of radix orifice, the conventional access opening maybe modified to a trapezoidal shape for accurate location of the orifice. ${ }^{4,6}$ In this case, both the above mentioned points were applicable. If the canal orifice is covered by secondary dentine, the secondary dentine is differentiated from the pulp chamber floor which is comparatively darker than the calcified dentine. ${ }^{4}$ In this case, the orifice was not completely covered with secondary dentine, however, it was narrow and therefore, flaring at orifice level was performed with gates glidden number 5 . NiTi instruments have the ability to maintain original canal 
curvature and produce a tapering root canal shape However, broken or separated instruments is one of the disadvantages of NiTi instruments. ${ }^{10}$ Two factors which play an important role in instrument fracture include cyclic fatigue and torsional fatigue. Torsional failure develops when friction between the instrument and root canal dentine leads to increased torque that exceeds the torsional strength of the instrument, resulting in deformation and fracture. ${ }^{12}$ Glide path is important to reduce torsional fatigue. This helps in increasing the root canal diameter atleast equal to the tip diameter of the rotary instrument which is used for shaping the canals. This in turn reduces the torsional stress on the instrument. ${ }^{13}$ The cyclic fatigue is dependent upon the curvature of root canal. Moreover, as the file rotates, it undergoes repeated cycles of tension and compression. This continuous cyclic load may result in crack commencement and ultimately fracture of instrument. ${ }^{8}$ As the angle of curvature of root canal increases, cyclic stress acting upon the instrument increases. On the other hand, the lesser the angle of curvature, the more the severity of curvature, the more cyclic stress placed on curvature and therefore, the more chances of instrument fracture. ${ }^{8}$ Studies have shown that an curvature in the coronal or middle third of the root canal is more likely to lead to failure compared to an apical curvature. ${ }^{14}$ In this case, a sharp curvature in the coronal portion of the accessory canal was felt while scouting with the small files. The curvature was suspected in buccolingual plane. Therefore, cyclic fatigue of the rotary files could be the cause of instrument fracture in this case.

Single use of all rotary NiTi instruments has been recommended. However, due to the increased cost of the rotary NiTi instruments, reuse of the files is always pertinent. However, studies have shown that prolonged use of NiTi instruments reduces their cyclic fatigue resistance. ${ }^{15,16} \mathrm{In}$ this case, reuse of the rotary PTU files could be a cause of their decreased cyclic fatigue resistance.

Several factors affect the instrument removal from the root canal. These include diameter, length and position of the fractured instrument and diameter, length and curvature of the root canal. ${ }^{10}$ Instruments that lie in the straightaway portions of the canal can typically be removed. In this case, the coronal portion of the fractured instrument was visible as the instrument was in coronal portion of the root canal, therefore an attempt to retrieve the separated fragment was planned.

A number of techniques have been described in different studies to remove fractured endodontic instruments from the canal. ${ }^{17-19}$ In this case, the technique for broken file removal, described by Cliff Ruddle was applied. In this technique, ultrasonic instruments are used for the removal of fractured instrument in conjunction with a microscope.
In this technique, coronal straight line access is achieved followed by creation of a staging platform on the coronal aspect of the separated instrument. This staging platform is created using modified gates glidden which works when activated in intimate contact with the separated instrument and moved in counterclockwise direction. The ultrasonic energy causes the separated instrument to unwind and sometimes jump out of the canal. ${ }^{10}$ In this case, coronal flaring was performed with gates glidden number 5 and 4 . Gates number 3 was modified to create a staging platform. Ultrasonic tip was then used for trephining, however, due to the short length of the ultrasonic tip and the unavailability of the specialized ultrasonic tip for Endodontics, modified gates glidden number 2 was used for the purpose of trephining. Till date, no such case has been reported in which modified gates glidden have been used for trephining.

\section{CONCLUSION}

Clinicians should be aware of these unusual root morphologies in the mandibular first molars. Preoperative identification of radix entomolaris or paramolaris is important to facilitate the Endodontic procedure. Use of CBCT and dental operating microscope are significant in detection of morphologies.

The most important factors central to successful instrument removal are knowledge, training, and competency in selecting the best presently developed and proven technologies and techniques. Importantly, no single removal method will always produce the desired result. As such, successful removal oftentimes requires patience, perseverance and creativity.

\section{CONFLICT OF INTEREST}

None declared

\section{REFERENCES}

1. Barker BCW, Parsons KC, Mills PR, Williams GL. Anatomy of root canals. III. permanent mandibular molars. Aust Dent J. 1974;19:40813.

https://doi.org/10.1111/j.1834-7819.1974.tb02372.x

2. Vertucci FJ. Root canal anatomy of the human permanent teeth. Oral Surg Oral Med Oral Pathol. 1984;58:589-99.

https://doi.org/10.1016/0030-4220(84)90085-9

3. Skidmore AE, Bjorndal AM. Root canal morphology of the human mandibular first molar. Oral Surg Oral Med Oral Pathol. 1971;32:778-84. https://doi.org/10.1016/0030-4220(71)90304-5

4. Abella F, Mercadé M, Duran-Sindreu F, Roig M. Managing severe 
curvature of radix entomolaris: three-dimensional analysis with cone beam computed tomography. Int Endod J. 2011;44:876-85. https://doi.org/10.1111/j.1365-2591.2011.01898.x

5. Štamfelj I. Who coined the term radix entomolaris? Int Endod J. 2014;47:810-11.

https://doi.org/10.1111/iej.12213

6. De Moor RJG, Deroose CAJG, Calberson FLG. The radix entomolaris in mandibular first molars: an endodontic challenge. Int Endod J. 2004;37:789-99.

https://doi.org/10.1111/j.1365-2591.2004.00870.x

7. Peters OA. Current challenges and concepts in the preparation of root canal systems: a review. J Endod. 2004;30:559-67.

https://doi.org/10.1097/01.DON.0000129039.59003.9D

8. Pruett JP, Clement DJ, Carnes DL. Cyclic fatigue testing of nickeltitanium endodontic instruments. J Endod. 1997;23:77-85. https://doi.org/10.1016/S0099-2399(97)80250-6

9. Endodontic diagnosis: American Association of Endodontists; 2013 [cited 2017]. Available from: https:/www.aae.org/specialty/wpcontent/uploads/sites/2/2017/07/endodonticdiagnosisfall2013.pdf.

10. Ruddle CJ. Nonsurgical Retreatment. J Endod. 2004;30:827-45. https://doi.org/10.1097/01.don.0000145033.15701.2d

11. Carlsen OLE, Alexandersen V. Radix entomolaris: identification and morphology. Eur J Oral Sci. 1990;98:363-73.

https://doi.org/10.1111/j.1600-0722.1990.tb00986.x

12. Blum JY, Machtou P, Ruddle C, Micallef JP. Analysis of Mechanical Preparations in Extracted Teeth Using ProTaper Rotary Instruments: Value of the Safety Quotient. J Endod. 2003;29:567-75. https://doi.org/10.1097/00004770-200309000-00007
13. Berutti E, Negro AR, Lendini M, Pasqualini D. Influence of Manual Preflaring and Torque on the Failure Rate of ProTaper Rotary Instruments. J Endod. 2004;30:228-30.

https://doi.org/10.1097/00004770-200404000-00011

14. Peters OA, Paque F. Current developments in rotary root canal instrument technology and clinical use: a review. Quintessence Int (Berlin, Germany : 1985). 2010;41:479-88.

15. Gambarini G. Cyclic Fatigue of Nickel-Titanium Rotary Instruments after Clinical Use with Low-and High-Torque Endodontic Motors. J Endod. 27:772-74.

https://doi.org/10.1097/00004770-200112000-00015

16. Plotino G, Grande NM, Sorci E, Malagnino VA, Somma F. A comparison of cyclic fatigue between used and new Mtwo Ni-Ti rotary instruments. Int Endod J. 2006;39:716-23.

https://doi.org/10.1111/j.1365-2591.2006.01142.x

17. Yang Q, Shen Y, Huang D, Zhou X, Gao Y, Haapasalo M. Evaluation of Two Trephine Techniques for Removal of Fractured Rotary Nickeltitanium Instruments from Root Canals. J Endod. 2017;43:116-20. https://doi.org/10.1016/j.joen.2016.09.001

18. Ormiga F, da Cunha Ponciano Gomes JA, de Araújo MCP. Dissolution of Nickel-Titanium Endodontic Files via an Electrochemical Process: A New Concept for Future Retrieval of Fractured Files in Root Canals. J Endod. 2010;36:717-20.

https://doi.org/10.1016/j.joen.2009.11.024

19. Terauchi Y, O'Leary L, Kikuchi I, Asanagi M, Yoshioka T, Kobayashi C, et al. Evaluation of the Efficiency of a New File Removal System in Comparison With Two Conventional Systems. J Endod. 2007;33:58588.

https://doi.org/10.1016/j.joen.2006.12.018 